Nephrologe 2014 · 9:195-195

DOI 10.1007/s11560-013-0809-1

Online publiziert: 15. Mai 2014

๑) Springer-Verlag Berlin Heidelberg 2014
H. Haller ${ }^{1}$ D.E. Müller-Wiefel ${ }^{2}$

${ }^{1}$ Klinik für Nieren- und Hochdruckerkrankungen, Medizinische Hochschule Hannover

2 Hamburg

\title{
Nierensteine - eine vielfach unterschätzte nephrologische Herausforderung
}

Nierensteine werden in der Regel dann diagnostiziert, wenn sie sich selbstständig machen und eine Akutsymptomatik verursachen, die zur urologischen Intervention führt. Somit stellt im klinischen Alltag die Urolithiasis eine Domäne der Urologen dar, mit der der Nephrologe sich zumeist eher weniger identifiziert - und das, obwohl Nierensteine mit einer wachsenden Prävalenz von 5\% schon jetzt nach der arteriellen Hypertonie die häufigste Erkrankung der Niere darstellen und darüber hinaus auch heute noch mit einem immensen Rezidivrisiko von etwa $60 \%$ belastet sind. Bei kompetenter nephrologischer Ursachendiagnostik, kombiniert mit angemessener Patientenführung unter Berücksichtigung der Adhärenzproblematik, dürfte eine deutlich erfolgreichere Metaphylaxe möglich sein, die die belastenden Leidenswege der steinbildenden Patienten ebenso optimieren mag wie die damit verbundenen nicht unerheblichen Kosten für unser Gesundheitssystem.

Diese Ausgabe von Der Nephrologe soll dazu beitragen, das Wissen auf dem Gebiet der Nephrolithiasis zu aktualisieren, indem auf die gegenwärtigen Kenntnisse zur Pathogenese der Nierensteine, allen voran der häufigsten aus Kalziumoxalat (ca. $75 \%$ ), und die heute zur Verfügung stehenden konservativen wie invasiven Therapiemöglichkeiten eingegangen wird.

Besonderes Augenmerk wird mit zwei Artikeln auf die primären, weil hepatogenetisch fixierten Hyperoxalurien gelegt, früher weniger erklärend als Oxalose bezeichnet. Es wird der dabei heute mögliche diagnostische Standard ebenso abge- handelt, wie auf die je nach Individualsituation einsetzbare Strategie der Nierenund/oder Lebertransplantation eingegangen wird, die wegen der Schwere der metabolischen Intoxikation und der daraus resultierenden Ohnmacht der Blutreinigungsverfahren zumeist schon das Kindes- und Jugendalter betrifft. Demzufolge ist zwar zunächst der pädiatrische $\mathrm{Ne}$ phrologe angesprochen, der aber im Verlauf bei sich weiter verbessernden Überlebenschancen dieser jungen Patienten vom internistischen Nephrologen in der Betreuung abgelöst wird. So mag die dargestellte Thematik der Hyperoxalurien vielleicht auch dazu beitragen, eine Verbesserung der sicher komplizierten Transition dieses außergewöhnlichen Patientenklientels von der pädiatrischen in die internistische Betreuung zu erzielen.

In diesem Sinne dürfen wir Ihnen $\mathrm{Mu}$ ße und Freude beim Studium der für Sie in diesem Themenheft zusammengestellten Lektüre wünschen.

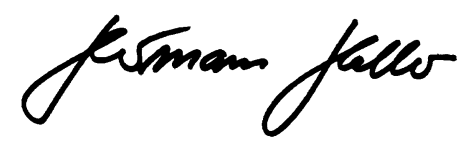

H. Haller

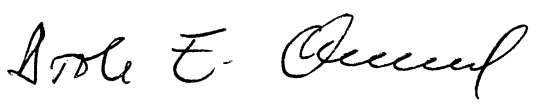

D.E. Müller-Wiefel

\section{Korrespondenzadressen}

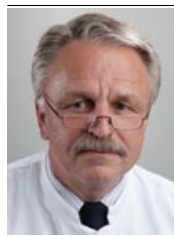

Prof. Dr. H. Haller

Klinik für Nieren- und Hochdruckerkrankungen, Medizinische Hochschule Hannover

Carl-Neuberg-Straße 1, 30625 Hannover haller.hermann@ mh-hannover.de

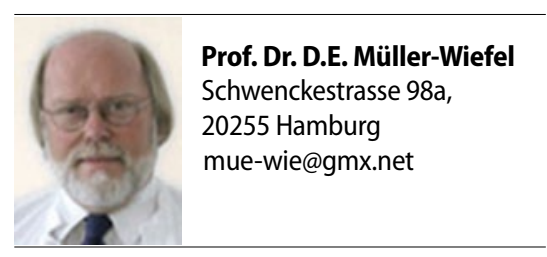

Interessenkonflikt. H. Haller und D.E. Müller-Wiefel geben an, dass kein Interessenkonflikt besteht. 